\title{
Simulation of an optically induced asymmetric deformation of a liquid-liquid interface
}

\author{
Hamza Chraïbi a,*, Didier Lasseux ${ }^{\mathrm{a}}$, Eric Arquis ${ }^{\mathrm{a}}$, \\ Régis Wunenburger ${ }^{\mathrm{b}}$, Jean-Pierre Delville ${ }^{\mathrm{b}}$, \\ ${ }^{a}$ Université Bordeaux I \\ Transferts, Écoulements, Fluides, Énergétique (TREFLE), UMR CNRS 8508 \\ Esplanade des Arts et Métiers \\ 33405 Talence Cedex, France. \\ ${ }^{\mathrm{b}}$ Université Bordeaux I \\ Centre de Physique Moléculaire Optique et Hertzienne (CPMOH), UMR CNRS \\ 5798 \\ 351 Cours de la Libération \\ 33405 Talence cedex, France.
}

\begin{abstract}
Deformations of liquid interfaces by the optical radiation pressure of a focused laser wave were generally expected to display similar behavior, whatever the direction of propagation of the incident beam.

Recent experiments showed that the invariance of interface deformations with respect to the direction of propagation of the incident wave is broken at high laser intensities. In the case of a beam propagating from the liquid of smaller refractive index to that of larger one, the interface remains stable, forming a nipple-like shape, while for the opposite direction of propagation, an instability occurs, leading to a long needle-like deformation emitting micro-droplets. While an analytical model successfully predicts the equilibrium shape of weakly deformed interface, very few work has been accomplished in the regime of large interface deformations. In this work, we use the Boundary Integral Element Method (BIEM) to compute the evolution of the shape of a fluid-fluid interface under the effect of a continuous laser wave, and we compare our numerical simulations to experimental data in the regime of large deformations for both upward and downward beam propagation. We confirm the invariance breakdown observed experimentally and find good agreement between predicted and experimental interface hump heights below the instability threshold.
\end{abstract}

Key words: Opto-hydrodynamics - Optical radiation pressure - Boundary integral element method - Interfacial flow.

Preprint submitted to European Journal of Mechanics B/Fluids 25 October 2018 


\section{Introduction}

The deformation of liquid-liquid interfaces by the optical radiation pressure has received increasing attention in the past few years as many practical applications of laser-induced surface deformation are now under development. Among others, we can cite interfacial characteristics measurements such as viscosity [1] or surface tension [2], as well as fluid membranes manipulation with optical tweezers [3; 4]. Effects of the radiation pressure have been also recognized as an appealing non-intrusive tool for local manipulation of liquid or soft materials giving birth to many applications in biotechnologies [5; 6].

Historically, the deformation of fluid-fluid interfaces resulting from the radiation pressure induced by an impinging focused laser beam was first identified in the early 70's by Ashkin and Dziedzic [7]. Later, Zhang \& Chang [8] illuminated a $50 \mu \mathrm{m}$ radius water drop with a $100-200 \mathrm{~mJ}$ laser pulse and showed strong distortion of the droplet surface at its front and rear regions. At low pulse energy, the droplet interface exhibited oscillations that damped out on time scales of tens of microseconds, whereas at high energy a disruption generating a jet of micro-droplets was observed at the rear part of the drop. The drop distortion was theoretically studied by Lai et al. [9] and later by Brevik et al. [10]. Based on a linear wave theory adapted to low energy pulses, their analysis predicted drop oscillations very similar to those observed in the experiments. However, drop deformations under higher energy pulses were not modelled as their linear model can no longer be used to describe the droplet shapes in the regime close to disruption.

Thus, current existing theoretical descriptions of optically induced flow and surface deformations are restricted to small amplitude deformations. However, recent experiments on very soft interfaces have evidenced several regimes, ranging from the so-called classical linear regime for small beam intensity, in which the height of the deformation linearly depends on the radiation to Laplace pressure ratio referred to in the following as $\xi$ [11], to nonlinear regimes [12; 13] with a possible interface breakup for even larger beam intensities [14]. In these recent experiments, a continuous $\mathrm{Ar}^{+}$laser wave (wavelength in vacuum $\lambda_{0}=514.5 \mathrm{~nm}$ ) of waist $\omega_{0} \approx 3$ to $15 \mu \mathrm{m}$ was used to bend the interface between two liquid phases in coexistence close to their liquid-liquid critical point. Two reasons motivated this choice. As the separated phases of these near-critical mixtures have very low surface tension $\left(\gamma \sim 10^{-7} \mathrm{~N} / \mathrm{m}\right)$, a laser beam of moderate power $P \sim 1 \mathrm{~W}$ becomes sufficient to induce large interface deformations of typical size $\sim 10-100 \mu \mathrm{m}$. Moreover, near-criticality raises universality concepts demonstrating the generality of the purpose. As

\footnotetext{
* Corresponding author.

Email address: h.chraibi@cpmoh.u-bordeaux1.fr (Hamza Chraïbi ).
} 
predicted from the photon momentum balance at the interface, in the linear regime the radiation pressure induces the same deformations for upward or downward laser propagation [11; 13]. However, in the nonlinear regime the invariance of the interface deformation with respect to the direction of propagation of the wave breaks down. Stable nipple-like deformations were observed in the case of a propagation from the less refractive fluid (marked as (1)) to the more refractive one (marked as (2)) (upward propagation) [12], while in the other case where the laser wave propagates from the more refractive fluid to the less refractive one (downward propagation), the interface becomes unstable, leading to the formation of a needle-like deformation emitting micro-droplets [14] (see Fig. 1).

(a)
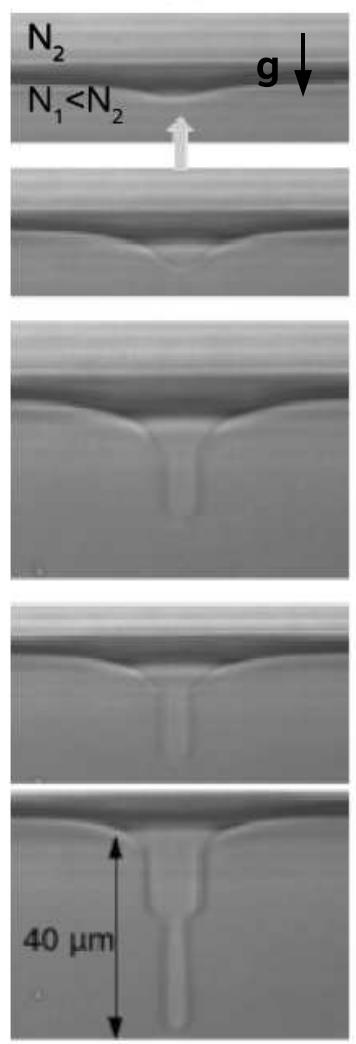

(b)
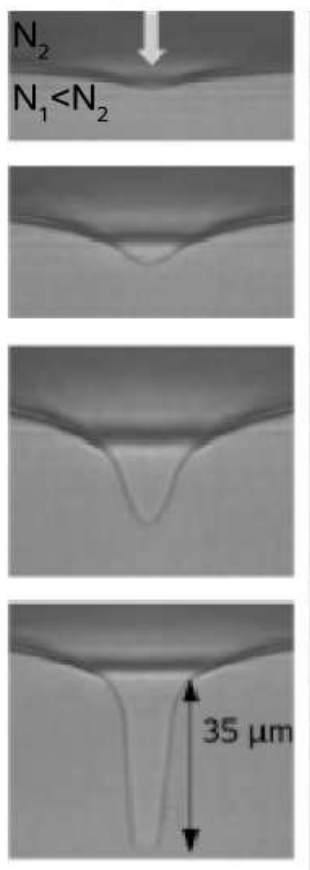

(c)

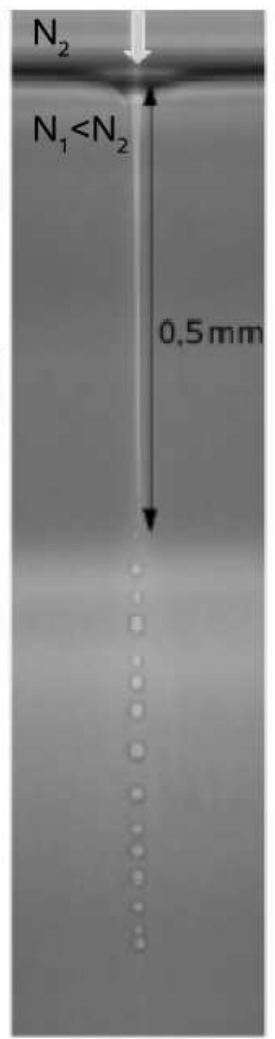

Fig. 1. Interface deformations induced at $\left(T-T_{C}\right)=3.5 \mathrm{~K}$ by a laser beam of waist $\omega_{0}=5.3 \mu \mathrm{m}$. (a) Laser propagating upward from the less refractive fluid to the more refractive fluid as indicated by the white arrow. $\mathrm{P}$ increases from top to bottom and is successively equal to $120,240,360,390$ and $720 \mathrm{~mW}$. (b) Downward direction of propagation. $\mathrm{P}=124,248$ and $372 \mathrm{~mW}$ from top to bottom; the bottommost picture $(405 \mathrm{~mW}$ ) shows the destabilization of the interface leading to the formation of a stationary jet similar to that illustrated in (c).

The aim of the present work is to investigate whether the differences in interface deformation with respect to the direction of propagation of the wave (called hereafter invariance breakdown) is numerically predictable and if the 
predicted interface shapes and heights agree with experiments. Our paper is structured as follows. In Section 2, we briefly describe the experimental setup, sample properties, and the physical model for laser-induced interface deformations. This model assumes an axisymmetric Stokes flow in each liquid and a boundary condition at the interface describing the competition between viscosity, optical radiation pressure, capillarity and gravity effects. A brief description of the Boundary Integral Element Method (BIEM) is presented in Section 3, emphasizing the advantages of its application to interfacial flows. In Section 4, comparisons between numerical and experimental results are shown and discussed for both the linear/nonlinear regimes of deformation and both directions of propagation, illustrating by the way the efficiency of our approach to investigate the subtle coupling between the effects of light and flow.

\section{Experimental configuration and physical model}

Exhaustive experimental details on the configuration and protocol used here were reported earlier $[11 ; 13]$.

\subsection{Experiments}

In figure 2 we have represented a picture of a typical liquid-liquid interface deformation induced by a laser beam propagating upward, together with the notations used throughout this work. Cylindrical coordinates $\left(\mathbf{e}_{\mathbf{r}}, \mathbf{e}_{\mathbf{z}}, \mathbf{e}_{\alpha}\right)$ with their origin $O$ located at the intersection of the beam axis with the initial flat interface are chosen for this study and are shown in Fig. 2. A point $\mathbf{x}$ is thus marked by the space coordinates $(r, z, \alpha)$.

Incidence and transmission angles of light are respectively denoted by $\theta_{i}$ and $\theta_{t}$.

The two-phase liquid sample is enclosed in a fused-quartz cell of optical path length $l=2 \mathrm{~mm}$. The bending of the liquid-liquid meniscus is driven by a linearly polarized continuous $A r^{+}$laser (wavelength in vacuum $\lambda_{0}=514.5 \mathrm{~nm}$ ) in the $\mathrm{TEM}_{00}$ gaussian mode. The beam is weakly focused on the interface by a $10 \times$ microscope objective to ensure a cylindrical distribution of the intensity near the meniscus. Thus, in the vicinity of the liquid-liquid interface, the laser beam intensity $I$ has the following distribution:

$$
I(r, z) \approx I(r)=\frac{2 P}{\pi \omega_{0}^{2}} \exp \left(-2\left(\frac{r}{\omega_{0}}\right)^{2}\right)
$$




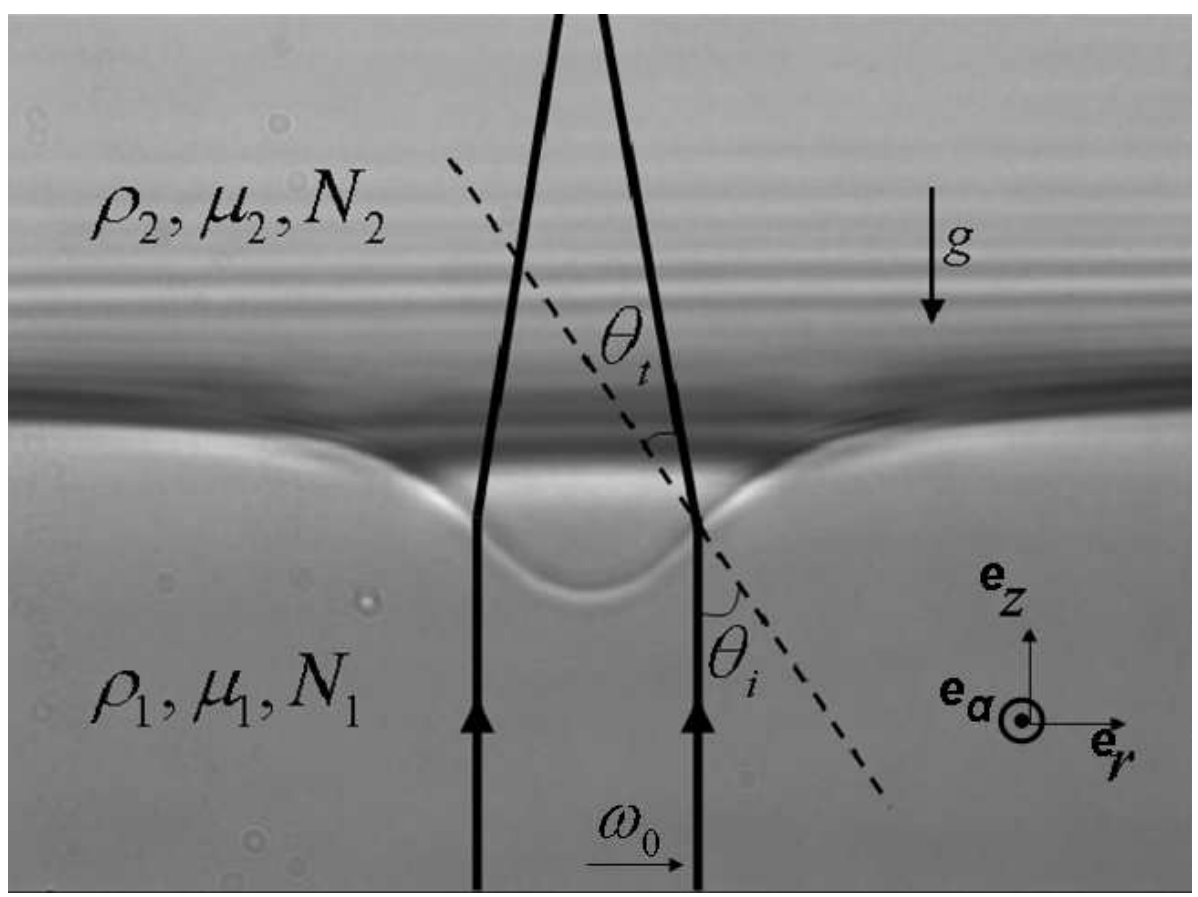

Fig. 2. Liquid-liquid interface deformation by a focused laser wave propagating upward. $T-T c=3.5 K ; \omega_{0}=5.3 \mu \mathrm{m} ; P=240 \mathrm{~mW}$. The laser light scattered towards the camera has been filtered. See text for notations.

where $P$ is the laser beam power. The beam waist $\omega_{0}$ can be adjusted from 3 to $15 \mu \mathrm{m}$. Depending on the setup configuration, the laser beam propagates vertically either upward from fluid (1) to fluid (2) or downward from fluid (2) to fluid (1).

\subsection{Two-phase sample properties}

The investigated fluid-fluid interface is obtained according to the following procedure. Using a quaternary liquid mixture made of toluene, sodium dodecyl sulfate, n-butanol and water, we prepare a water-in-oil micellar phase of microemulsion whose composition is such that at room temperature it is close to a critical consolute line. Close to the liquid-liquid critical temperature, $T_{C}=308 \mathrm{~K}$, the critical thermodynamic behavior of the mixture belongs to the universality class $(\mathrm{d}=3 \mathrm{n}=1)$ of the Ising model [15]. For a temperature $T>T_{C}$ it separates in two micellar phases of different concentrations $\varphi_{1}$ and $\varphi_{2}$. The use of a near-critical two-phase fluid is motivated by the fact that significant interface deformations by electromagnetic radiation, without nonlinear propagation effects [15] or disturbing thermal coupling, require weak interfacial tension and buoyancy. With our fluids, both effects are fulfilled since they vanish when the critical point is neared. Moreover, the interfacial tension $\gamma$ of phase-separated near-critical supra molecular fluids is intrinsi- 
cally smaller than that of near-critical pure fluids, thus enhancing even more interface deformations.

The mixture is thermally controlled at a temperature $T$ above $T_{C}$. Since the density (index of refraction) of water is larger (smaller) than that of toluene, the micellar phase of largest concentration $\varphi_{1}$, density $\rho_{1}$ and smallest refractive index $N_{1}$ is located below that of lower micellar concentration $\varphi_{2}$, density $\rho_{2}$ and of refractive index $N_{2}$. Given the temperature range investigated in the reported experiments $T-T_{C}=2$ to $15 \mathrm{~K}$, thermophysical properties of the two-phase sample can be satisfactorily evaluated using usual asymptotic scaling laws of near-critical phenomena. For interfacial tension:

$$
\gamma=\gamma_{0}\left(\frac{T-T_{c}}{T_{c}}\right)^{2 \nu}
$$

with $\gamma_{0}=10^{-4} \mathrm{~N} / \mathrm{m}$ and $\nu=0.63$. Assuming that (i) a scaling law accurately describes the variations of $\Delta \varphi=\varphi_{1}-\varphi_{2}$ in the investigated broad temperature domain $T-T_{C}$ and that (ii) the coexistence curve is symmetric versus the critical concentration $\varphi_{c}$, the micellar concentration in each phase can be estimated by:

$$
\begin{aligned}
& \varphi_{1}=\varphi_{c}+\frac{\Delta \varphi}{2} \\
& \varphi_{2}=\varphi_{c}-\frac{\Delta \varphi}{2}
\end{aligned}
$$

with $\varphi_{c}=0.11$ and:

$$
\Delta \varphi=\Delta \varphi_{c}\left(\frac{T-T_{c}}{T_{c}}\right)^{\beta}
$$

with $\beta=0.325$.

The value of the critical amplitude $\Delta \varphi_{c}$, can be estimated theoretically for this system with the main assumption that our mixture is binary $\Delta \varphi_{c}=$ $\sqrt{16 \pi \varphi_{c} R^{+}}=1.458[16 ; 17] . R^{+}=0.37$ being a universal ratio in microemulsions [18]. As micellar phases are in fact quaternary components fluids, their phase diagram presents some asymmetry in $\varphi$ leading either to over or underestimate the critical amplitude $\Delta \varphi_{c}$ depending on which side of the coexistence curve is chosen. The theoretical value of $\Delta \varphi_{c}$ leads to an overestimation of the optical index contrast $\Delta N$. Consequently a modified value for $\Delta \varphi_{c}, 0.42$, has been adopted in this study, based on a quantitative comparison between numerical and experimental interface steady hump heights in the linear regime of deformations. This modified value gives acceptable predictions for the con- 
centrations $\varphi_{1}$ and $\varphi_{2}$. In fact, $\Delta \varphi_{c}$ should be understood as a free parameter in the estimation of phases properties.

The density of each phase $\rho_{i}, i=1,2$ can be written as a function of $\varphi_{i}$ :

$$
\rho_{i}=\rho_{m i c} \varphi_{i}+\rho_{\text {cont }}\left(1-\varphi_{i}\right)
$$

where $\rho_{\text {mic }}=1045 \mathrm{~kg} \cdot \mathrm{m}^{-3}$ and $\rho_{\text {cont }}=850 \mathrm{~kg} \cdot \mathrm{m}^{-3}$ are the densities of the micelles and continuous phases respectively. At $T-T_{C}=3.5 \mathrm{~K}$ the estimated density contrast is $\Delta \rho=\rho_{1}-\rho_{2}=\simeq 20 \mathrm{~kg} / \mathrm{m}^{3}$.

As the average distance between two micelles is small compared to the wavelength of the laser wave, the mixture can be regarded as homogeneous from the electromagnetic point of view. Thus, the mean-field model for the relative dielectric permittivity $\epsilon_{i}$ of the mixture predicts [21]:

$$
\epsilon_{i}\left(\varphi_{i}\right)=\varphi_{i} \epsilon_{m i c}+\left(1-\varphi_{i}\right) \epsilon_{c o n t}-\frac{\varphi_{i}\left(1-\varphi_{i}\right)\left(\epsilon_{m i c}-\epsilon_{c o n t}\right)^{2}}{3\left(\varphi_{i} \epsilon_{m i c}+\left(1-\varphi_{i}\right) \epsilon_{c o n t}\right)} .
$$

This relation is used to estimate $N_{i}$ taking into account the fact that:

$$
\epsilon_{i}=N_{i}^{2}, i=1,2 .
$$

along with $\epsilon_{m i c}=1.86$ and $\epsilon_{\text {cont }}=2.14, \epsilon_{\text {mic }}$ and $\epsilon_{\text {cont }}$ being the relative permittivity of the micelles and continuous phases respectively.

At $T-T_{C}=3.5 K$, the optical indices are $N_{1}=1.447$ and $N_{2}=1.457$.

In addition, since concentrations are weak, we use Einstein's relation to estimate the dynamic viscosity $\mu_{i}$ of each phase:

$$
\begin{aligned}
& \mu_{1}=\mu_{0}\left(1+2.5 \frac{\Delta \varphi}{2}\right) \\
& \mu_{2}=\mu_{0}\left(1-2.5 \frac{\Delta \varphi}{2}\right),
\end{aligned}
$$

with $\mu_{0}=1.269$ Pa.s.

Nevertheless, these values could be shifted by possible laser heating of the fluids. In order to estimate the resulting change in the physical properties such as interfacial tension $\gamma$ or viscosity $\mu_{i}$ due to temperature increase, we consider the steady diffusion equation in cylindrical coordinate with the absorbed laser intensity as a source term:

$$
\nabla^{2} T_{I}(r)+\frac{\alpha_{t h}}{\Lambda_{t h}} I(r)=0
$$


We assume stationary conditions since the thermal diffusion time scale is much smaller than the viscous one. $T_{I}(r)$ is the local increase of temperature due to local heating of the laser wave. $\alpha_{t h} \simeq 310^{-4} \mathrm{~cm}^{-1}$ is the thermal absorption and $\Lambda_{t h}=1.2810^{-3} \mathrm{Wcm}^{-1} \mathrm{~K}^{-1}$ is the thermal conductivity. Using a FourierBessel transform to solve equation (11) [19; 16], we find that the maximum increase in temperature is:

$$
T_{I}(r=0) \simeq \frac{\alpha_{t h} P}{4 \pi \Lambda_{t h}} \ln (100 \Gamma)
$$

where $\Gamma=1.781$ is the Euler constant. Considering now equation (2), we can estimate the change of interfacial tension due to the increase in temperature:

$$
\frac{\partial \gamma}{\partial T}=\gamma \frac{1.26}{T-T_{C}}
$$

At $T-T_{C}=3.5 K$ and for $P=1 W$, we find that $\frac{\partial \gamma}{\partial T} \simeq 1.310^{-7} N^{-1} K^{-1}$ and $T_{I}(r=O) \simeq 0.1 K$ which leads to $\frac{\Delta \gamma}{\gamma} \simeq 3.6 \%$. Thermocapillary effects can thus be confidently discarded.

Considering now the viscous dependance on temperature, we use the following empirical law given for microemulsions [20]:

$$
\mu(T)=[1.934-0.019(T-273)] 10^{-3} .
$$

Consequently, $\frac{\partial \mu}{\partial T}=-0.01910^{-3}$ and thus for $P=1 W$ we find $\frac{\Delta \mu}{\mu} \simeq 2 \%$. This second estimation ensures negligible thermal effects.

\subsection{Electromagnetic force and pressure}

As expressed in [21], the total electromagnetic force per unit volume exerted by the laser in each phase is given by:

$$
\mathbf{f}_{\mathbf{e m} i}=-\frac{1}{2} \epsilon_{0} \mathbf{E}_{\mathbf{i}}^{2} \nabla \epsilon_{i}+\frac{1}{2} \epsilon_{0} \nabla\left[\mathbf{E}_{\mathbf{i}}^{2} \rho_{i} \frac{\partial \epsilon_{i}}{\partial \rho_{i}}\right]+\frac{\epsilon_{i}-1}{c^{2}} \frac{\partial}{\partial t}\left(\mathbf{E}_{\mathbf{i}} \times \mathbf{H}_{\mathbf{i}}\right) .
$$

In this expression, $\mathbf{E}$ (respectively $\mathbf{H}$ ) is the electric (respectively magnetic) field associated to the laser wave, $\epsilon_{0}$ is the permittivity of vacuum and $c=$ $310^{8} \mathrm{~m} / \mathrm{s}$ is the celerity of light in vaccum. 
The first term, whose jump across the liquid interface results in the usually called optical radiation pressure, is due to the change in photon momentum from one fluid to the other. This momentum change is due to the discontinuity of permittivities across the interface. As both fluids are assumed to be homogeneous, the optical radiation term cancels within each phase and only acts on the interface.

The second term, referred to as the electrostrictive force, also undergoes a jump at the interface due to the difference in both the optical properties and the electric fields $\mathbf{E}$ between the two liquids in contact. However, this force also acts as a bulk force within each phase because of the radial dependence of the electric field $\mathbf{E}$. We will demonstrate, in adequation with previous theoretical investigations [9; 10], that the electrostriction does not contribute to the motion and shape of the interface as its bulk contribution is compensated by its surface one. The quantity $\frac{\partial \epsilon_{i}}{\partial \rho_{i}}$, which depends on the density of each phase through a nonlinear relationship can be deduced from equations (6) and (7).

Finally, in equation (15), the third term is called the Abraham term, and is undetectable at optical frequencies [9; 10] and thus cancels out for our purpose.

In the following, we denote $E^{2}=<E^{2}>$ the quadratic value of the electric field averaged over an optical period.

Assuming incompressible fluids ( $\rho_{i}$ is homogeneous throughout each phase $i$, $i=1,2$ ), we can include contributions of the bulk forces (gravity and electrostriction) in the pressure field and define a pseudo-pressure $p_{i}$ given by:

$$
p_{i}=p_{i 0}+\rho_{i} g z-\frac{1}{2} \epsilon_{0}\left(E_{i}^{2} \rho_{i} \frac{\partial \epsilon_{i}}{\partial \rho_{i}}\right), i=1,2,
$$

where $p_{i 0}, i=1,2$, is the pressure in each phase and $g=9.81 \mathrm{~m} \cdot \mathrm{s}^{-1}$ is the acceleration of gravity.

The electromagnetic stress tensor defined by Landau [21] such that $\nabla \cdot \mathbf{T}_{\mathbf{i}}^{\mathrm{em}}=\mathbf{f}_{\mathrm{emi}}$ can be expressed as:

$$
\mathbf{T}_{\mathbf{i}}^{\mathbf{e m}}=\frac{1}{2} \epsilon_{0}\left(E_{i}{ }^{2} \rho_{i} \frac{\partial \epsilon_{i}}{\partial \rho_{i}}\right) \mathbf{I}-\frac{1}{2} \epsilon_{0} \epsilon_{i} E_{i}{ }^{2} \mathbf{I}+\epsilon_{0} \epsilon_{i} \mathbf{E}_{\mathbf{i}} \mathbf{E}_{\mathbf{i}}, i=1,2 .
$$

In the case of a steady interface, the jump from fluid 1 to fluid 2 of the local pressure and of the electromagnetic stress are balanced by the Laplace pressure:

$$
\left(p_{20}-p_{10}\right) \mathbf{n}+\left[\mathbf{T}_{\mathbf{1}}^{\mathbf{e m}}-\mathbf{T}_{\mathbf{2}}^{\mathbf{e m}}\right] . \mathbf{n}=\gamma \kappa(r) \mathbf{n},
$$


where $\mathbf{n}$ is the unit vector directed from liquid 1 to liquid 2 and normal to the interface and $\kappa(r)=\frac{1}{r} \frac{d}{d r} \frac{r z^{\prime}}{\sqrt{1+z^{\prime 2}}}$ is the curvature of the interface in cylindrical coordinates, $z^{\prime}=\frac{d z}{d r}$ is the local slope of the interface.

Rewriting Eq. (18) in terms of pseudo-pressures leads to:

$$
\begin{aligned}
&\left(p_{2}-p_{1}\right)+\left(\rho_{1}-\rho_{2}\right) g z+ \frac{1}{2} \epsilon_{0}\left(E_{2}{ }^{2} \rho_{2} \frac{\partial \epsilon_{2}}{\partial \rho_{2}}-E_{1}{ }^{2} \rho_{1} \frac{\partial \epsilon_{1}}{\partial \rho_{1}}\right) \\
&+\mathbf{n} \cdot\left[\mathbf{T}_{\mathbf{1}}^{\mathbf{e m}}-\mathbf{T}_{\mathbf{2}}^{\mathbf{e m}}\right] \cdot \mathbf{n}=\gamma \kappa(r) .
\end{aligned}
$$

Therefore, the bulk contribution of the electrostriction is balanced by its surface contribution and the interface shape does not depend on electrostriction. Consequently, the equilibrium equation of the interface can be written as:

$$
\left(p_{2}-p_{1}\right)+\left(\rho_{1}-\rho_{2}\right) g z-\frac{1}{2} \epsilon_{0}\left(\epsilon_{1} E_{1}{ }^{2}-\epsilon_{2} E_{2}{ }^{2}\right)+\epsilon_{0}\left(\epsilon_{1} \mathbf{E}_{\mathbf{1}} \mathbf{E}_{\mathbf{1}} \cdot \mathbf{n}-\epsilon_{2} \mathbf{E}_{\mathbf{2}} \mathbf{E}_{\mathbf{2}} \cdot \mathbf{n}\right) \cdot \mathbf{n}=\gamma \kappa(r) .
$$

At final equilibrium, only normal stress act on the interface, consequently there is no flow within the phases and this equilibrium in each phase is characterized by:

$$
\nabla p_{i}=0, i=1,2
$$

In the experiments, the laser wave was linearly polarized so that the electric field $\mathbf{E}=E \mathbf{e}_{\alpha}$ was perpendicular to the plane of observation of azimuthal coordinate $\alpha=0$. Therefore $\mathbf{E}$ is continuous across the interface in this plane.

Conversely, in the plane defined by $\alpha=\pi / 2$, the electric field is within the plane of propagation (parallel polarization) and thus is no longer continuous across the interface. However, as shown in [12], the first term of the electromagnetic force, which is responsible for the optical radiation pressure acting on the interface, is quasi-independent of beam polarization as long as refractive indices are sufficiently close to each others $\left(\frac{N_{1}}{N_{2}} \sim 1\right)$. We can then assume as in a previous investigation [22] a circular polarization of the electric field $\mathbf{E}$. Therefore, using the definition of the irradiance $I(r)=\frac{\epsilon_{0}}{2} N_{i} c E_{i}^{2}$ in the case of a beam propagating upward, the radiation pressure can be written as:

$$
\Pi^{u p}\left(r, \theta_{i}, \theta_{t}\right)=-\frac{I(r)}{c} \cos \theta_{i}\left(2 N_{1} \cos \theta_{i}-T^{u p}\left(N_{1} \cos \theta_{i}+N_{2} \cos \theta_{t}\right)\right),
$$


while in the case of a downward beam propagation it is given by:

$$
\Pi^{\text {down }}\left(r, \theta_{i}, \theta_{t}\right)=\frac{I(r)}{c} \cos \theta_{i}\left(2 N_{2} \cos \theta_{i}-T^{\text {down }}\left(N_{2} \cos \theta_{i}+N_{1} \cos \theta_{t}\right)\right)
$$

Here, $T^{u p}$ and $T^{\text {down }}$ are the Fresnel transmission coefficients in energy. In the case of a circular polarization, they are expressed as:

$$
\begin{aligned}
& T^{u p}=T^{\text {down }}=\left(2 N_{1} N_{2} \cos \theta_{i} \cos \theta_{t}\right) \\
& \left(\frac{1}{\left(N_{1} \cos \theta_{i}+N_{2} \cos \theta_{t}\right)^{2}}+\frac{1}{\left(N_{2} \cos \theta_{i}+N_{1} \cos \theta_{t}\right)^{2}}\right)
\end{aligned}
$$

As a final remark, note that the expression of the electromagnetic pressure in the case of a downward propagation is valid until achieving the condition of total reflection $\theta_{i}<\theta_{T R}=\arcsin \left(\frac{N_{1}}{N_{2}}\right)$. What occurs when light is totally reflected by a highly deformed interface is out of the scope of the present numerical study.

\subsection{Flow equations and boundary conditions}

We consider a cylindrical domain of radius $R$ and height $H$ as depicted in Figure 3. We choose to treat all the governing equations in a dimensionless form by using the laser waist $\omega_{0}$ as the characteristic length scale and the viscous relaxation velocity $u^{*}=\gamma / \mu_{2}$ as the reference velocity associated to the characteristic timescale $\tau^{*}=\mu_{2} \omega_{0} / \gamma$. The reference pressure is taken as $p^{*}=\frac{\mu_{i} u^{*}}{\omega_{0}}, i=1,2$. Since the weak absorption of light at laser frequency ensures negligible thermal effects, we consider all liquid properties $\left(\gamma, \rho_{i}, \mu_{i}\right.$, $i=1,2$ ) constant in adequation with our estimation of the change in interfacial tension and viscosity due to a local increase in temperature. Moreover, given the value of the Reynolds number of the flow $\rho_{2} u^{*} \omega_{0} / \mu_{2} \sim 10^{-3}$, inertia is negligible compared to viscous forces, allowing a quasi-steady creeping flow assumption. Thus, flow in each liquid is governed by Stokes and mass conservation equations respectively given by:

$$
\begin{aligned}
-\nabla p_{i}+\nabla^{2} \mathbf{u}_{\mathbf{i}} & =0 \\
\nabla \cdot \mathbf{u}_{\mathbf{i}} & =0, i=1,2 .
\end{aligned}
$$

$\mathbf{u}_{\mathbf{i}}$ is the dimensionless velocity in fluid $\mathrm{i}$ and $p_{i}$ is the dimensionless value of the pseudo-pressure defined in Eq.(16). The hydrodynamic divergence free stress tensor $\mathbf{T}_{\mathbf{i}}$ is:

$$
\mathbf{T}_{\mathbf{i}}=-p_{i} \mathbf{I}+\left(\nabla \mathbf{u}_{\mathbf{i}}+{ }^{t} \nabla \mathbf{u}_{\mathbf{i}}\right)
$$


According to Eq. (16), the normal stress jump across the interface $S_{I}$, which is compensated by gravity, optical radiation pressure and capillarity forces can be expressed in the following form:

$$
\left(\lambda \mathbf{T}_{\mathbf{1}} \cdot \mathbf{n}-\mathbf{T}_{\mathbf{2}} \cdot \mathbf{n}\right) \cdot \mathbf{n}=\kappa(r)-\Pi(r)-\text { Bo } z .
$$

In this equation, $\lambda=\mu_{1} / \mu_{2}$ is the viscosity ratio and $\mathrm{Bo}_{0}=\left(\rho_{1}-\rho_{2}\right) g \omega_{0}^{2} / \gamma$ is an optical Bond number which represents the ratio of gravity to capillary forces, $\Pi(r)$ is the dimensionless expression of the optical radiation pressure resulting from equations (22) and (23). We denote $\Pi(r)=\Pi(r)^{u p} \omega_{0} / \gamma$ for the upward direction of propagation and $\Pi(r)=\Pi(r)^{\text {down }} \omega_{0} / \gamma$ for the downward direction. In order to quantify the effects of the laser wave on the interface, we define $\xi$ as the electromagnetic to Laplace pressure ratio on beam axis at normal incidence:

$$
\xi=\Pi\left(r=0, \theta_{i}=0, \theta_{t}=0\right)=\frac{4 P}{\pi c \omega_{0} \gamma} \frac{N_{1}\left(N_{2}-N_{1}\right)}{\left(N_{2}+N_{1}\right)} .
$$

A no-slip condition at the interface along with the fact that fluids are immiscible implies continuity of the velocity $\mathbf{u}$ on the interface $S_{I}$ :

$$
\mathbf{u}=\mathbf{u}_{1}=\mathbf{u}_{\mathbf{2}} \text { for } \mathbf{x} \in S_{I}
$$

Moreover, we assume the classical no-slip boundary condition on the lateral, upper and lower solid walls $S_{C 1}$ and $S_{C 2}$ (see figure 3). This leads to:

$$
\mathbf{u}_{\mathbf{i}}=\mathbf{0} \text { for } \mathbf{x} \in S_{C i}, i=1,2 \text {. }
$$

In order to reproduce the experimental configuration, $R$ must be large enough compared to $\omega_{0}$ to meet the hypothesis of an infinite extent in the horizontal direction. Several tests were performed with different values of the dimensionless radius $\beta=\frac{R}{\omega_{0}}$ of the computational domain, and a value $\beta=70$ was found to be large enough to satisfy this constraint. The interface motion is obtained using a Lagrangian approach. It consists in tracking each fluid particle on the interface in its Lagrangian motion according to:

$$
\frac{d \mathbf{x}}{d t}=\mathbf{u}(\mathbf{x}) \text { for } \mathbf{x} \in S_{I}
$$

That is, the interface is advected along with the flow until equilibrium is reached for which velocities normal to the interface are zero, i.e. $\mathbf{u}(\mathbf{x}) \cdot \mathbf{n}=\mathbf{0}$ for $t \rightarrow t_{\infty}$. 


\section{Numerical method}

A brief description of the numerical algorithm is presented in this section. For more extensive details on the Boundary Integral Element Method (BIEM) applied to two-phase axisymmetric flow, the reader may refer to the review by Tanzosh et al. on the solution of free surface flow problems using this technique [23]. The BIEM reveals to be an excellent tool to solve interfacial flow problems with high resolution as reported in the analysis of flow involving electric and magnetic fields [24] or buoyancy [25; 26].

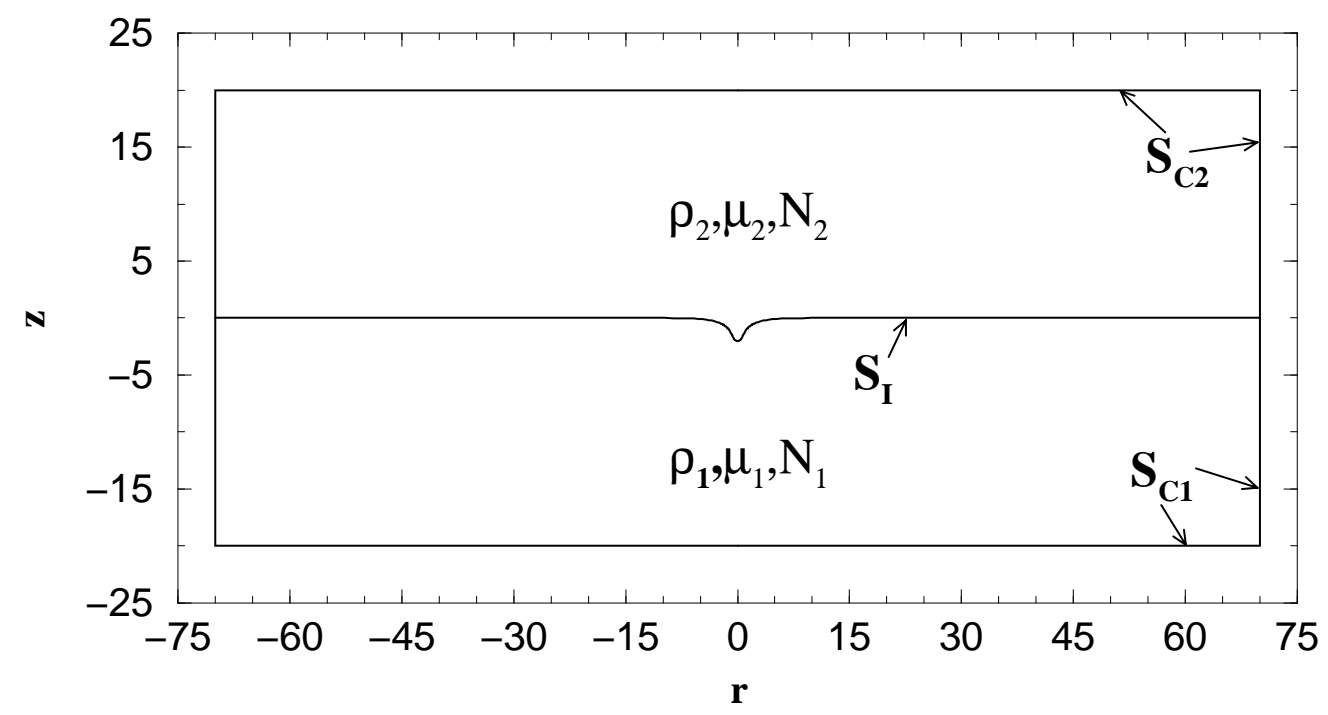

Fig. 3. Typical configuration of the computational domain showing the two fluids and the liquid interface. The domain is assumed to be axisymmetric along $\mathbf{e}_{\mathbf{z}}$.

Because solutions to the Stokes problem can be formulated in terms of Green's functions [27], we can rewrite the governing equations as a system of integral equations over the boundaries of the computational domain. Once boundary conditions on $S_{I}, S_{C 1}$ and $S_{C 2}$ are used, the two-phase Stokes problem can be written in the following compact form:

$$
\begin{aligned}
& \frac{1+\lambda}{2} \mathbf{u}(\mathbf{x})=\int_{S_{I}} \mathbf{U} \cdot \mathbf{n}\left(\kappa\left(r_{y}\right)-\Pi\left(r_{y}\right)-B o z\left(r_{y}\right)\right) d S_{y}+ \\
& (1-\lambda) \int_{S_{I}} \mathbf{n} . \mathbf{K} . \mathbf{u} d S_{y}+\lambda \int_{S_{C 1}} \mathbf{U} \cdot\left(\mathbf{T}_{\mathbf{1}} \cdot \mathbf{n}\right) d S_{y}-\int_{S_{C 2}} \mathbf{U} \cdot\left(\mathbf{T}_{\mathbf{2}} \cdot \mathbf{n}\right) d S_{y} .
\end{aligned}
$$

Here, $\mathbf{U}$ and $\mathbf{K}$ are Green kernels for velocity and stress respectively and are given by [27]: 


$$
\begin{aligned}
& \mathbf{U}(\mathbf{d})=\frac{1}{8 \pi}\left(\frac{1}{d} \mathbf{I}+\frac{\mathbf{d d}}{d^{3}}\right), \\
& \mathbf{K}(\mathbf{d})=-\frac{3}{4 \pi}\left(\frac{\mathbf{d d d}}{d^{5}}\right),
\end{aligned}
$$

where $\mathbf{d}=\mathbf{x}-\mathbf{y}, \mathbf{y}\left(r_{y}, z_{y}\right)$ is the integration point. In Eq. (33), the first term in the right hand side describes the flow contribution from interfacial tension, radiation pressure and gravity, whereas the second term accounts for shear rates contrast on the interface. This term vanishes when $\lambda=1$. The third and fourth terms account for shear occurring on $\left(S_{C 1}\right)$ and $\left(S_{C 2}\right)$ as a result of the no-slip boundary condition.

Velocities on the interface as well as stress over all the boundaries $S_{I}, S_{C 1}$ and $S_{C 2}$ are determined by solving the discrete form of this equation using a numerical procedure. This procedure requires first the discretization of all the boundaries $S_{I}, S_{C 1}$ and $S_{C 2}$. Due to integral formulation and axial symmetry, the problem is reduced to one dimension and only line boundaries, as represented in Figure 3, need to be discretized. In this work, the mesh is made of constant boundary elements i.e. line segments with centered nodes. The fluidfluid interface $S_{I}$ is parameterized in terms of arc length and is approximated by local cubic splines, so that the curvature can be accurately computed. Distribution and number of points are adapted to the shape of the interface, so that the concentration of elements is higher in regions where the variation of curvature of the interface is large. The number of mesh points is about 70 for a typical computation of a small interface deformation. The solid boundaries $S_{C 1}$ and $S_{C 1}$ are meshed using about 40 uniformly distributed points. An increase in the mesh resolution for the interface and the solid boundaries do not show any significant change in the results.

Azimuthal integration of Eq. (33) is performed analytically [28; 29] reducing Eq. (33) to a line integration which is finally performed using Gauss quadratures [30]. Elliptic integrals resulting from the azimuthal integration are evaluated using power series expansions [31].

The motion of the interface is followed using the kinematic condition (32) which is discretized using an explicit first-order Euler time scheme. A typical computation begins with a flat interface at rest. The laser beam is switched on at $t=0$, and the interface deforms towards fluid 1 of smallest refractive index.

Computation stops when an equilibrium state is reached $\left(\frac{d z_{(r=0, t)}}{d t} \rightarrow 0\right)$. The time step is chosen to be about 20 times smaller than $\tau^{*}$.

\section{Results and discussion}

In this section, we compare equilibrium hump heights and interface shapes obtained experimentally to their numerical predictions in both linear and non- 
linear regimes and for both directions of propagation. We also present the dynamics of hump formation expected in the nonlinear regime for both upward and downward propagations.

As mentioned before, few work were dedicated to the modelling of optical deformations of liquid interfaces in the nonlinear regime. Hallanger et al. [22] used a finite difference method to solve the equation describing the equilibrium state of the interface for a laser wave propagating from the fluid of smallest refractive index (here fluid 1) and Wunenburger et al. [13] used a simple model where gravity was neglected (Bo $<<1$ ) to predict the equilibrium hump heights in the nonlinear regime.

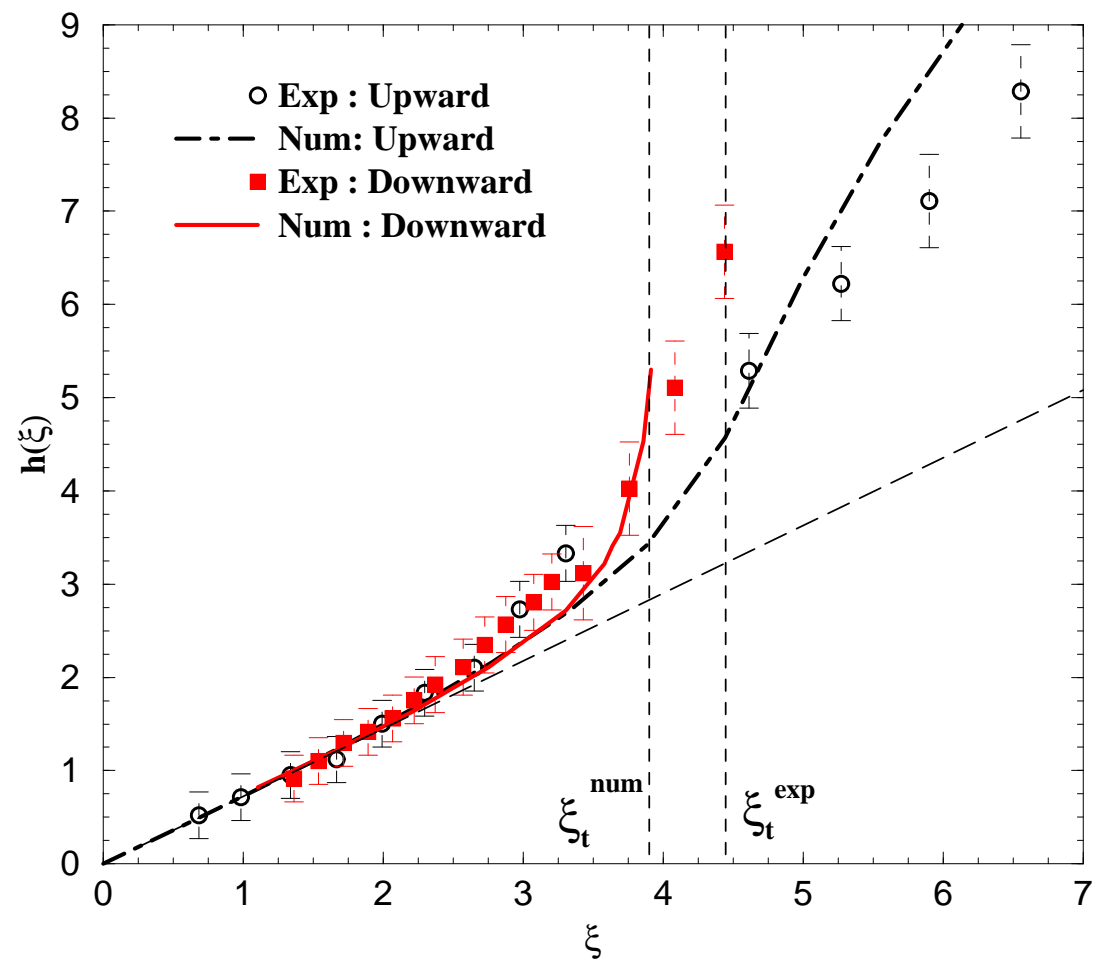

Fig. 4. Variation of the reduced hump height $h=|z(r=0)|$ versus the reduced pressure ratio $\xi$ when light propagates upward and downward for $\omega_{0}=5.3 \mu \mathrm{m}$ and $T-T c=3.5 K(B o=0.015)$. Comparison is made between experimental results (symbols) and the numerical resolution (lines). The bold dashed line is the prediction for the linear model. $\xi_{t}^{e x p}$ and $\xi_{t}^{\text {num }}$ respectively represent the experimental and numerical thresholds of instability in the case of downward propagation.

In Figure 4, we have represented the variation of the dimensionless equilibrium interface hump height $h$ as a function of the pressure ratio $\xi$ for upward and downward propagations. Experiments (symbols) and numerical predictions (lines) are shown together. 


\subsection{Steady-state deformations, linear regime}

Previous experiments $[11 ; 32$ performed in the linear regime of deformations showed that, for $\xi<2.5, h$ varies linearly with $\xi$ for both directions of propagation (symbols) and that the linear variation of $h(\xi)$ does not depend on the direction of propagation for $N_{1} / N_{2} \simeq 1$. A linear theory assuming $N_{1} \simeq N_{2}$ was proposed [11; 13] which quantitatively agree with these observations. Indeed, the linear theory gives:

$$
z(r)=-\frac{\xi}{4} \int_{0}^{\infty} \frac{e^{-k^{2} / 8}}{B o+k^{2}} J_{0}(k r) k d k
$$

where $J_{0}$ is the zeroth-order Bessel $J$ function. We deduce:

$$
h(r=0)=\frac{\xi}{8} e^{\frac{B o}{8}} E_{1}\left(\frac{B o}{8}\right)
$$

where $E_{1}(x)$ is the one-argument exponential function $\left[E_{1}(x)=\int_{x}^{\infty} \frac{e^{-k}}{k} d k\right]$. In the version published in European Journal of Mechanics, there is an error on the equation (37) where $h(r=0)=\frac{\xi}{2} \ldots$. This is corrected in this postprint.

When $N_{1}$ and $N_{2}$ are calculated using Eqs. (77) and (8), we find that the predicted $h(\xi)$ variation perfectly agrees with experiments for both directions of propagation when $\xi<2.5$ (i.e. in the linear regime), as shown in Fig. 4 in the particular case $\omega_{0}=5.3 \mu \mathrm{m}, T-T_{C}=3.5 \mathrm{~K}$, i.e. Bo $=0.015$ (dashed straight line). As shown in Fig. 4, the numerical predictions $h(\xi)$ for both upward (dot-dashed line) and downward propagations (solid line) are also found to agree with both the linear theory and experiments. In addition, Fig. 5 shows good agreement between the experimental interface shape (dark symbols) and its prediction using both the linear theory (open square) and the numerical simulation (solid line) at $\xi=1.35$ for both the upward (Fig. 5a) and the downward propagation (Fig. 5b). This validates the numerical code versus linear analytical predictions as well as its accuracy versus experimental results.

\subsection{Steady-state deformations, nonlinear regime}

Experimentally, for $\xi \geq 2.5, h(\xi)$ gradually deviates from linearity. For an upward propagation (open circles in Fig. 4) $h(\xi)$ increases with a larger slope than that predicted by the linear theory when $2.5 \leq \xi<3$.4. For larger values of $\xi, h(\xi)$ increases again nonlinearly with a slope lying between that of the linear regime and the above mentioned nonlinear one. 
Conversely, in the case of downward propagation (dark squares), $h$ increases with $\xi$ up to $\xi \simeq 3.4$, where its slope diverges. The divergence of $h^{\prime}(\xi)$ leads to the jetting instability studied in details in $[13 ; 14 ; 32]$ and illustrated in Fig. 1 .

The invariance breakdown of the hump height below the instability threshold can be qualitatively explained by considering how the electromagnetic pressure $\Pi\left(r, \theta_{i}, \theta_{t}\right)$ varies with the incidence angle $\theta_{i}$ for both propagations [12]. Whereas the electromagnetic pressure, which varies as $\frac{\cos \theta_{i}}{\cos \theta_{t}}$, monotonously decreases with $\theta_{i}$ in the case of upward propagation because $\theta_{t}=\arcsin \left(\frac{N_{1}}{N_{2}} \sin \theta_{i}\right)$, conversely it continuously increases with $\theta_{i}$ in the case of downward propagation because $\theta_{t}=\arcsin \left(\frac{N_{2}}{N_{1}} \sin \theta_{i}\right)$, as shown in the inset in Fig. 6. Thus, a downward propagating laser beam exerts an electromagnetic pressure on a strongly deformed interface (along which $\sin \theta_{i}$ can reach values comparable to unity) larger than an upward propagating beam. The jetting instability occurring for the downward propagation can be explained by the fact that the beam propagates in this case from the large to the low refractive fluid which makes total reflection of light at the interface achievable [13; 14].

The comparison between numerical simulation results and experimental data in the nonlinear regime indicates that, in the case of the upward propagation, a satisfactory agreement is found regarding $h(\xi)$ (see Fig. 4) for the experimentally investigated values of $\xi$. Moreover, good agreement regarding the interface shape is observed up to $\xi \simeq 5$, as shown in Fig. 5a for the particular value of $\xi=2.9$ and $\xi=4.6$. This represents a noticeable progress, since previous work on the subject [22] qualitatively reproduced interface shapes (in the case of upward propagation) but did not compare experiments to numerical predictions.

For $\xi$ larger than 3.5, nipple-like interface shapes, shown in Fig. 1, are experimentally observed. They are not reproduced by the numerical simulations suggesting an additionnal coupling or feedback effect between the exciting beam and the soft interface that is not investigated in the present work. As also shown in Fig. 4, in the case of downward propagation, the numerical simulation qualitatively reproduces the experimentally observed monotonous behavior of $h(\xi)$ up to the jetting instability occurring at $\xi=\xi_{t}^{\text {num }}$, as well as the divergence of its slope at the instability threshold. Instability of the interface is numerically predicted to occur when the wave undergoes total reflection and is a priori focused toward the hump tip, i.e. when $\theta_{i}$ reaches $\theta_{T R}$ at the inflection point of the interface. As a matter of fact, since total reflection and focusing of the incident wave by the interface is assumed to be responsible for the jetting instability [12], we assume that beyond $\xi_{t}^{\text {num }}$ the interface will actually become unstable. Moreover, beyond $\xi_{t}^{n u m}$ the physical model used in the numerical model fails, in particular due to Eq. (23) because the numerical simulation of the jet formation and stability should include the description of 
the complex interplay between wave propagation and interface deformation acting as a wave guide, which is beyond the scope of the present investigation. Still, the discrepancy between the values of $\xi_{t}^{\text {num }}$ at which total reflection occurs along the interface $\left(\xi_{t}^{n u m}=3.9\right.$ in Fig. 4$)$ and $\xi_{t}^{e x p}$ just beyond which the bell-shaped interface actually loses its stability $\left(\xi_{t}^{\exp }=4.43\right.$ in Fig. 4$)$ calls for a study of the interface shape.
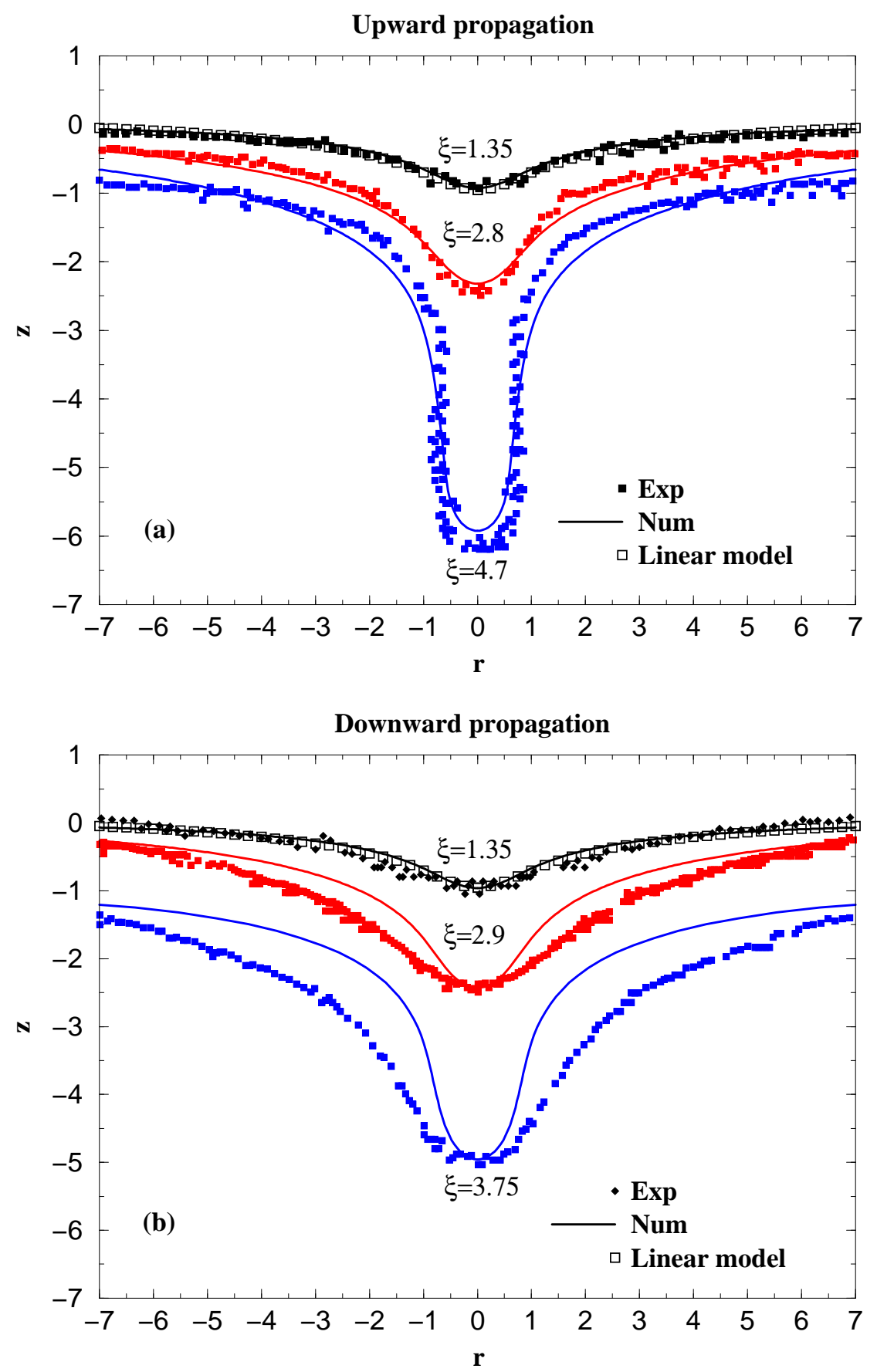

Fig. 5. Comparison between experimental and numerical interface profiles for upward (a) and downward (b) propagations. $\omega_{0}=5.3 \mu \mathrm{m}$ and $T-T c=3.5 K(B o=0.015)$. 
Figure 5b shows that there is also a noticeable discrepancy between the experimental and numerically predicted interface shapes for the case of the downward propagation in the nonlinear regime. More precisely, the experimental hump is wider than the numerically predicted one, and the experimental slope of the interface shape is smaller than the numerically predicted one all along the interface, even when the experimental hump height is well predicted numerically, as it is the case for $\xi=3.75$ and shown in Fig. 5b. This observation can explain the discrepancy observed on $h(\xi)$ obtained experimentally and predicted numerically. As a matter of fact, since the actual slope of the interface shape is all along the interface smaller than numerically predicted, the actual incidence angle $\theta_{i}$ is also smaller than that numerically predicted. Consequently, the total reflection of the wave by the interface reaching a slope equal to $\theta_{T R}$ and the associated jetting instability are expected to experimentally occur at a value of $\xi$ larger than that numerically predicted.

Nevertheless, the reason for this discrepancy between the actual interface shape and its numerical prediction in the case of a downward propagation is still unexplained. In Ref. [13] several possible causes were discussed and discarded. Among them are the effect of thermocapillary flows due to light absorption, the role of optical nonlinearities, and the additional electromagnetic pressure applied on the hump tip due to the partial reflection of light by the interface below the instability threshold. A more probable cause may be the viscous stress applied on the interface by the flow induced by the scattering of light as a result of refractive index inhomogeneities occurring in the bulk of each phase [33]. Indeed, the resulting scattering force is known (i) to be oriented in the direction of propagation and (ii) of radial extension much larger than the beam diameter. However, the exact effect of such a flow on finite size humps, in particular the interplay between viscous effects on the deformed interface and the feed back of the hump shape on the flow field in its vicinity, remains misunderstood and calls for further study.

\subsection{Dynamics of hump formation, nonlinear regime}

Previous work has recently shown that in the linear regime the interface dynamics is accurately described by a linear theory of overdamped interfacial circular waves [32], and that the interface dynamics is independent of the direction of propagation.

However, in the nonlinear regime, the interface dynamics actually depends on the wave propagation.

In Fig. 6, the time evolution of the hump height $h(t)$ is plotted for both the upward (dot-dashed line) and downward propagations (solid line) for $\xi=$ 


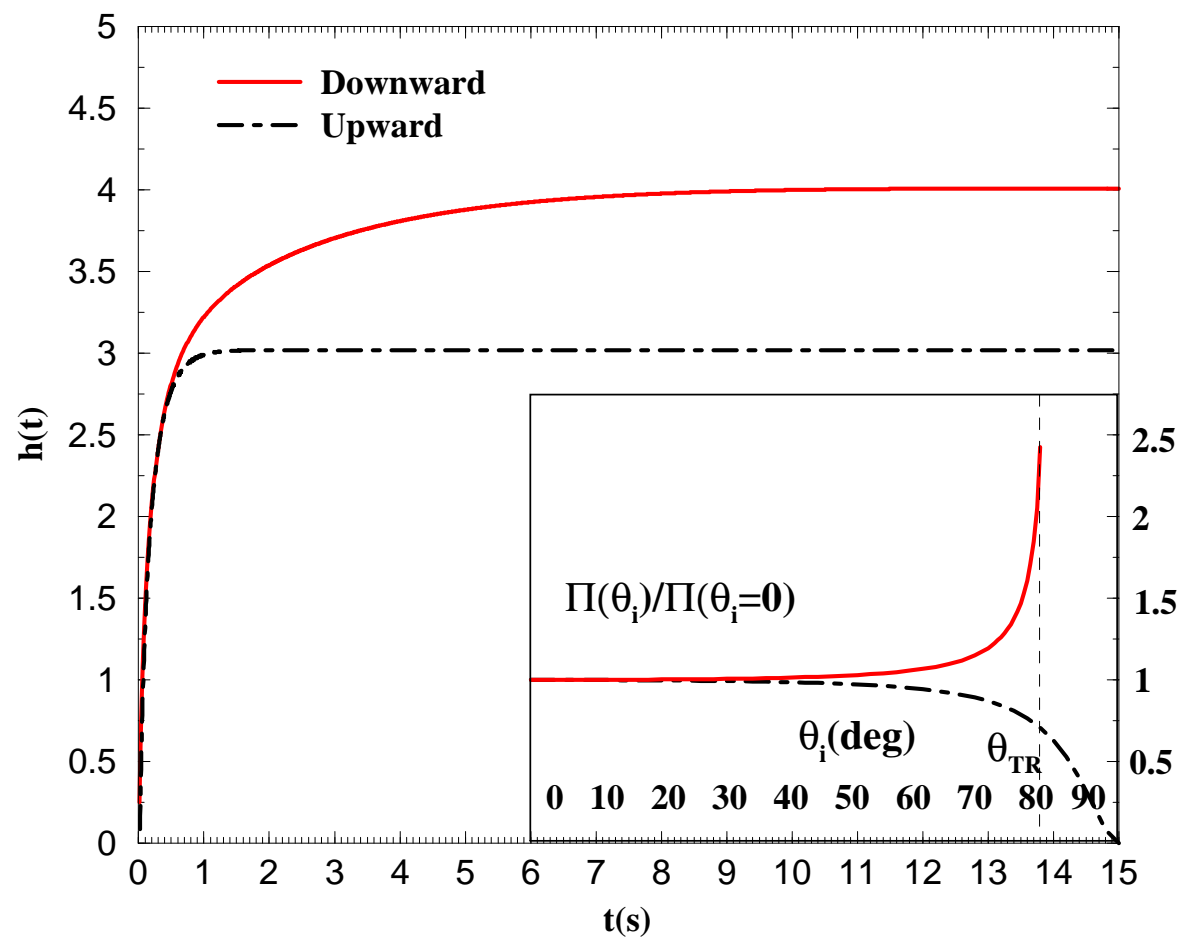

Fig. 6. Time evolution of the interface hump height for downward and upward propagation. $\omega_{0}=5.3 \mu \mathrm{m}, T-T c=3.5 K(B o=0.015)$ and $\xi=3.75$. Time $\mathrm{t}$ is dimensional. The inset shows the variation of the radiation pressure normalized by its value at normal incidence, versus the incidence angle for both propagations. The dashed line (inset) shows the total reflection threshold $\theta_{T R}$.

3.75 , a value belonging to the nonlinear regime. We note that (i) both curves coincide during the first $500 \mathrm{~ms}$ of the dynamics and eventually separates, (ii) the transient is significantly shorter in the case of upward propagation and the equilibrium state is reached much faster. The first observation can be explained by noting that for $t<500 \mathrm{~ms}$ both interface deformations are small enough to be described by a linear theory which is precisely independent of the beam propagation. The second observation can be qualitatively explained by considering the increase of the electromagnetic pressure with the incidence angle $\theta_{i}$ in the case of downward propagation (as shown in the inset of Fig. 6). As $\theta_{i}$ increases along the interface the associated overpressure applying on the growing hump increases its final height and thus increases the delay for the hump to reach equilibrium. On the contrary, the fact that the electromagnetic pressure decreases when $\theta_{i}$ increases in the case of an upward propagation leads to the opposite conclusion. Consequently, the analysis of the dynamics shows that, beyond the regime of linear deformations, our numerical approach becomes predictive for the complex nonlinear regime, thus calling for new experimental investigation and providing indications on how to perform these experiments. 


\section{Conclusions}

A Boundary Integral Element Method has been used to simulate the deformation of a fluid-fluid interface induced by a focused laser beam. Since interface deformations with azimuthal invariance were expected, an axisymmetric model has been developed. Comparisons between numerical predictions and experimental data showed two main results. First, our model can satisfactorily reproduce the variations of interface equilibrium hump height $h$ with respect to the ratio of electromagnetic to capillary pressure $\xi$ in regimes of small as well as of large deformations. In the case of an upward propagation, nipple-like interface shapes were not reproduced, while in the case of a downward propagation, instability threshold was overestimated probably because actual interface shapes are wider than numerically predicted. This could be due to bulk steady flows induced by light scattering as a result of liquid index inhomogeneitiy which deserves further experimental characterization before numerical implementation. A study of the dynamics of the interface for both directions of propagation was also performed showing a shorter characteristic time for the interface reaching its steady state in the case of an upward propagation. Future work should take into account the total reflection and focusing of light by the deformed interface in order to potentially predict hydrodynamic instability leading to jet formation and micro-droplet emission at the tip. Another interesting investigation would be to study the effect of light scattering induced flow [33] on jetting instability. Our first results are thus very encouraging since they illustrate the accuracy of our numerical model to describe and predict intriguing properties of nonlinear behaviors of the coupling between light and liquid interfaces, a subject of increasing interest due to its wide range of applications in soft matter physics on the one hand and scarce theoretical results on the other hand.

\section{Acknowledgements}

This research was supported by Centre National de la Recherche Scientifique (France), Université Bordeaux 1, and Conseil Régional d’Aquitaine.

\section{References}

[1] Y. Yoshitake, S. Mitani , K. Sakai and K. Takagi, Measurement of high viscosity with laser induced surface deformation technique, J. Appl. Phys., 97, (2005), 024901.

[2] S. Mitani and K. Sakai. Measurement of ultralow interfacial tension with a laser interface manipulation technique. Phys. Rev. E, 66, (2002), 031604.

[3] C. L. Guo, C. H. Lee, and J.Wang. Optical measurement of the viscoelastic 
and biochemical responses of living cells to mechanical perturbation. Opt. Lett., 23, (1998) ,307-309.

[4] W. C. Lin, C. H. Lee, and J.Wang. All-optical measurements of the bending rigidity of lipid-vesicle membranes across structural phase transitions. Phys. Rev. E, 64, (2001), 020901.

[5] J. Guck, R. Ananthakrishnan, T. J. Moon, C. C. Cunningham, and J. Käs. Optical deformability of soft biological dielectrics. Phys. Rev. Lett., $84(2000), 5451-5454$.

[6] E. Moses, R. Bar-ziv, and P. Nelson. Dynamic excitations in membranes induced by optival tweezers. Biophys. J., 75 (1998), 294-320.

[7] A. Ashkin and J. M. Dziedzic. Radiation pressure on a free liquid surface. Phys. Rev. Lett., 30, (1973), 139-142.

[8] J. Z. Zhang and R. K. Chang. Shape distorsion of a single water droplet by laser induced electrostriction. Opt. Lett., 13, (1988), 916-918.

[9] H. M. Lai, P. T. Leung, K. L. Poon, and K. Young. Electrostrictive distortion of a micrometer-sized droplet by a laser pulse. J. Opt. Soc. Am. B, 6 (1989), 12.

[10] I. Brevik and R. Kluge. Oscillations of a water droplet illuminated by a linearly polarized laser pulse. J. Opt. Soc. Am. B, 16:976-985, 1999.

[11] A. Casner and J. P. Delville. Giant deformations of a liquid-liquid interface induced by the optical radiation pressure. Phys. Rev. Lett., 87, (2001), 054503.

[12] A. Casner, J. P. Delville, and I. Brevik. Asymmetric optical radiation pressure effects on liquid interfaces under intense illumination. J. Opt. Soc. Am. B, 20, (2003), 11.

[13] R. Wunenburger, A. Casner, and J. P. Delville. Light-induced deformation and instability of a liquid interface. i. statics. Phys. Rev. E, 73 (2006), 036314 .

[14] A. Casner and J. P. Delville. Laser-induced hydrodynamic instability of fluid interfaces. Phys. Rev. Lett., 90, (2003), 14.

[15] E. Freysz, E. Lafon, J. P. Delville, and A. Ducasse. Phase conjugation in critical microemulsions. Phys. Rev. E, 49, (1994) 2141.

[16] A. Casner. Déformation, manipulations et instabilités d'interfaces liquides induites par la pression de radiation d'une onde laser. PhD thesis, Université Bordeaux I, (2002).

[17] J. S. Langer and A. J. Schwartz. Kinetics of nucleation in near-critical fluids. Phys. Rev. A. 21 ,(1980), 948-958.

[18] K. K. Mon. New Monte Carlo estimates of critical interfacial amplitudes and the universality of amplitude ratios. Phys. Rev. Lett. 60(26),(1988), 2749-2752.

[19] R. S. Moore and S. P. S. Porto and J. P. Gordon and K. C. C. Leite and J. R. Whinnery. Long-transient effects in lasers with inserted liquid samples. J. Appl. Phys. 30(1), (1965) , 3-8.

[20] E. Freysz. Etude des non-linéarités optiques dans les mélanges liquides binaires critiques. Phd. Thesis. Université Bordeaux 1 ,(1990). 
[21] L. D. Landau and E.M. Lifshitz. Electrodynamics of continuous media. Pergamon, Oxford, (1946).

[22] A. Hallanger, I. Brevik, and S. Haaland. Nonlinear deformations of liquidliquid interfaces induced by electromagnetic radiation pressure. Phys. Rev. E, 71, (2005), 056601, .

[23] J. Tanzosh, M. Manga, and H. A. Stone. Boundary integral method for viscous free-boundary problems: Deformation of single and multiple fluidfluid interfaces. Boundary Element Technology, VI:19-39, (1992).

[24] J. D. Sherwood. Breakup of fluid droplets in electric and magnetic fields. J. Fluid Mech., 188, (1987), 133-146.

[25] M. Manga and H. A. Stone. Low Reynolds number motion of bubbles, drops and rigid spheres through fluid-fluid interfaces. J. Fluid Mech., 287, (1994), 279-298, .

[26] D. M. Koch and D. L. Koch. Numerical and theoretical solutions for a drop spreading below a free fluid surface. J. Fluid Mech., 287, (1994), 251278.

[27] C. Pozrikidis. Boundary integral and singularity methods for linearized viscous flow. Cambridge text in applied mathematics, (1992).

[28] S. H. Lee and L. G. Leal. The motion of a sphere in the presence of a deformable interface. J. Fluid Mech., 87, (1982), 81-106.

[29] G. Graziani. A boundary integral function method for axisymmetric viscous flows. Int. Engng Sci., 27-7, (1989), 855-864.

[30] P. J. Davis and P. Rabinowitz. Methods of numerical integration. Academic Press, (1984).

[31] A. A. Bakr. The boundary integral equation method in axisymmetric stress analysis problems. Lecture Notes in Engineering, (1985).

[32] R. Wunenburger, A. Casner, and J. P. Delville. Light-induced deformation and instability of a liquid interface. ii. dynamics. Phys. Rev. E, 73, (2006), 036315.

[33] R. Schroll, R. Wunenburger, A. Casner, W. Zhang, and J.P. Delville. Liquid transport due to light scattering. Phys. Rev. Lett., 98, (2007) , 133601. 